\title{
Prevalence of Dentulism, Partial Edentulism and Complete Edentulism in Rural and Urban Population of Malwa Region of India: A Population-based Study
}

\author{
${ }^{1}$ Subhash Sonkesariya, ${ }^{2}$ Deshraj Jain, ${ }^{3}$ Prabha Shakya, ${ }^{4}$ Rohit Agrawal, ${ }^{5}$ SV Sai Prasad
}

\begin{abstract}
Background: The objective of this study was to evaluate the prevalence of dentulism, partial edentulism and complete edentulism in rural and urban population of Malwa region of India, who visited at department of prosthodontics for the first time.
\end{abstract}

Materials and methods: An institution-based, cross-sectional study using the random sampling method was used to select the study sample of 1000 males and females. Data were collected using questionnaires and oral examination. Data were statistically analyzed using Chi-square test.

Results: Nearly, 38.5 and $63.1 \%$ of the rural and urban females were dentulous in the age of below 53 and 43 years. 61.4 and $36 \%$ of rural and urban males were dentulous in the age of below 53 and 43 years. Nearly, 41.9 and $48.2 \%$ of the rural and urban females were partial edentulous in the age of above 53 and 43 years. Fifty-eight and $51.7 \%$ of rural and urban males were dentulous in the age of above 53 and 43 years. Nearly, 22.4 and $55.8 \%$ of the rural and urban females were completing edentulous in the age of above 43 years in both population. 77.5 and $44.1 \%$ of rural and urban males were completing edentulous in the age of above 33 and 53 years.

Limitation: The study was conducted in an institution-based set-up. Hence, any interpretation of the results of this study must bear this limitation in mind.

Conclusion: It is essential to identify feasible strategies to provide primary dental health education and treatment to all rural and urban elderly in the future. We suggest community dental health services to be included in general health of the elderly rather than a special health need of the community.

\footnotetext{
${ }^{1,4}$ Senior Lecturer, ${ }^{2}$ Professor and Head, ${ }^{3}$ Practicing Doctor ${ }^{5}$ Principal Scientist

1,2Department of Prosthodontics, Government Dental College Indore, Madhya Pradesh, India

${ }^{3}$ Department of Prosthodontics, Prathmesh Dental Hospital Navi Mumbai, Maharashtra, India

${ }^{4}$ Department of Public Health Dentistry, College of Dental Sciences and Research, Bhilai, Chhattisgarh, India

${ }^{5}$ Department of Agriculture, Indian Agricultural Research Institute-Regional Station, Indore, Madhya Pradesh, India

Corresponding Author: Subhash Sonkesariya, Senior Lecturer Department of Prosthodontics, Government Dental College Indore, Madhya Pradesh, India, Phone: 09920697449, e-mail: prathmeshdcc@gmail.com
}

Keywords: Tooth loss, Dentulism, Partial edentulism, Complete edentulism, Prevalence.

How to cite this article: Sonkesariya $S$, Jain $D$, Shakya $P$, Agrawal R, Prasad SVS. Prevalence of Dentulism, Partial Edentulism and Complete Edentulism in Rural and Urban Population of Malwa Region of India: A Population-based Study. Int J Prosthodont Restor Dent 2014;4(4):112-119.

Source of support: Nil

Conflict of interest: None

\section{INTRODUCTION}

Edentulism and a small number of remaining teeth are associated with low education level and family income. ${ }^{1}$ Edentulism is defined as the loss of permanent teeth and is the treatment outcome of a multifactorial process involving biologic process (dental caries, periodontal disease, trauma and others) as well as nonbiologic factors related to dental procedures (access to care, patient preferences). ${ }^{2}$ The prevalence and patterns of tooth loss have been studied to a certain extent in western countries and a few such studies have been carried out in India. ${ }^{1-8}$ Dental caries and periodontitis are caused by microorganisms, but age, gender, oral hygiene and lifestyle (tobacco smoking, alcohol intake) may modify the progression of these disorders. The influence of socioeconomic and the sociodemographic factors on edentulousness have been well-documented. ${ }^{1-9}$

World Health Organization databanks indicate that dental caries is prevalent in the majority of countries internationally with some reporting $100 \%$ incidence in their population, severe periodontal disease is estimated to affect 5 to $20 \%$ of the population and the incidence of complete edentulism has been estimated between 7 and $69 \%$ internationally. ${ }^{1}$ Several cross-sectional studies on the prevalence of edentulousness show consistently that edentulousness is associated with age, gender and living areas in most countries. ${ }^{1,2,4,9}$

Edentulism rates among the elderly have been reported as relatively high in number of European countries, such as England (74-79\%), Scotland (85\%), Ireland (72\%), Northern Ireland (69\%), Netherlands (83\%), Denmark (68\%), Finland (67\%) and Norway (57\%). In Australia, 68\% 
of people aged 65 or more were edentulous. Edentulism is consistently shown to increase with age, with females having higher rates of edentulism than do males. ${ }^{4}$

We do not have any statistics of proportion of loss of teeth or percentage of the population of India who are dentulous, partial edentulous and completely edentulous, so this study was carried out in both rural and urban population of Malwa region of India.

\section{AIMS AND OBJECTIVES}

The present study was conducted with the following aims and objectives.

- To evaluate the prevalence of dentulism, partial edentulism and complete edentulism in relation to age, gender, socioeconomic and sociodemographic factors.

\section{MATERIALS AND METHODS}

The Malwa region occupies a plateau in western Madhya Pradesh and south-eastern Rajasthan (between $21^{\circ} 10^{\prime} \mathrm{N}$ $73^{\circ} 45^{\prime} \mathrm{E}$ and $25^{\circ} 10^{\prime} \mathrm{N} 79^{\circ} 14^{\prime} \mathrm{E}$ ), with Gujarat in the west. The region includes the Madhya Pradesh districts of Agar, Dewas, Dhar, Indore, Jhabua, Mandsaur, Neemuch, Rajgarh, Ratlam, Shajapur, Ujjain, and parts of Guna and Sehore, and the Rajasthan districts of Jhalawar and parts of Banswara and Pratapgarh. The population of the Malwa region stood at 18.9 million in 2001, with a population density of a moderate $231 / \mathrm{km}^{2}$. The annual birth rate in the region registered 31.6 per 1000 , and the death rate 10.3. The infant mortality rate reported at 93.8, slightly higher than the overall rate for the Madhya Pradesh state.

An institution-based, cross-sectional, descriptive, epidemiological study on randomly selected population to determine the prevalence of dentulous, partial edentulous and complete edentulous patients in relation to age, gender, socioeconomic and sociodemographic factors reporting to the Government Dental College, Indore (MP), for the first time was conducted. A total of 1000 subjects, who reported to Government Dental College and Hospital, Indore (MP), were selected. Ethical clearance was obtained prior to the survey from the Ethics Committee of Government Dental College and Hospital, Indore. The informed consent of each patient was taken prior to recording oral health. Subjects were selected on the basis of oral examination only, not by radiographic examination. The questionnaire was finally prepared and coded with the help of dental research council epidemiological unit. The criteria used for selection of subjects in the study were: (1) Individuals should be $>13$ years, (2) only permanent dentition was considered, (3) third molar was excluded in the study and (4) individuals selected in the appropriate age groups by random sampling.

\section{Questionnaire Proforma}

A self-prepared questionnaire, which sought inquiries related to sociodemographic factors, which included name, age, gender, education, occupation, monthly income, reasons of edentulism (trauma, dental caries, periodontal disease, tobacco smoking, alcohol intake, diabetes, hypertension, heart failure) was prepared, but for the convenience we only included dental caries, trauma, periodontitis and others. Next part of the questionnaire elicited information regarding duration of edentulism and reasons of edentulism.

\section{PROCEDURE FOR COLLECTION OF DATA}

The questionnaire was completed personally for each patient who agreed to participate in the study, in the form of an interview, which appeared like normal conversation to allow for introduction and exploration of ideas and probe more deeply. Following the completion of the interview, the patients signed at the end of the questionnaire to mark their consent.

\section{STATISTICAL ANALYSIS}

The collected data were statistically analyzed. Descriptive statistics was applied and Pearson's Chi-square test was used to test variables. The collected data were analyzed statistically using the Chi-square test at the significance level of $\mathrm{p}<0.05$.

\section{Chi-square Test}

It is a nonparametric test, used when data were expressed in frequency or proportion or percentages. Chi-square test is used to evaluate the statistical significance of differences in frequencies between subgroups. Chi-square checks the difference between observed and expected values. The formula used for Chi-square test is:

Where,

$\chi^{2}=\frac{\Sigma(\mathrm{O}-\mathrm{E})^{2}}{\mathrm{E}}$

Where,

$\Sigma$-denotes summation

$\mathrm{O}$ - observed frequency

E- expected frequency

$\mathrm{p}$-value denotes level of significance:

$\mathrm{p}>0.05$, nonsignificant

$\mathrm{p}<0.05$, significant

$\mathrm{p}<0.01$, highly significant (significant at $99 \%$ confidence level)

$\mathrm{p}<0.001$, very highly significant (significant at $99.9 \%$ confidence level). 


\section{RESULTS}

India is a country of more than one billion people comprising number of religions, casts and sociodemographic variances. It is practically difficult to conduct nationwide epidemiological studies on dentulism, partial edentulism and complete edentulism. There is also lack of broader epidemiological studies on prevalence of tooth loss. Scant and scattered data on edentulism are available in few rural and urban areas among Indian population..$^{10-18}$ The quality and design of studies varies considerably, therefore, it is difficult to make a direct comparison.

Out of 1000 subjects, $362(36.2 \%)$ were from rural region and $638(63.8 \%)$ were from urban region. Out of 512 male subjects, 224 (43.7\%) were from rural and 288 (56.2\%) were from urban region. Out of 488 female subjects, 138 $(28.2 \%)$ were from rural and $350(71.7 \%)$ were from urban region (Table 1).

In urban population, a significant difference $(<0.01)$ was observed for dentulous nature in between the female and male subjects as well as for different age groups $(<0.05)$. According to the region, out of 179 female subjects, 49 subjects were from the rural region and 130 subjects were from the urban region. More females (130) were dentulous as compared to males (76) when subjects above 43 years, and subjects below 43 years in both females and males were dentulous. Among the subjects interviewed and examined, there were no dentulous subjects in both males and females in the age group of above 53 years (Table 1). Whereas, in rural population, a significant difference $(<0.05)$ was observed for dentulous nature between the female and male subjects, and nonsignificant difference between the different age groups of 13 to 52 years. It was observed that more males (78) were dentulous as compared to females (49), and subjects below 53 years in both female and male were more dentulous. Among the subjects interviewed and examined, there were no dentulous subjects in both males and females in the age group of above 53 years. In total population, nonsignificant difference (at par) was observed between the female and males subjects for dentulous nature as well as in between different age groups. Among the subjects interviewed and examined, there were no dentulous subjects in both males and females in the age group of above 53 years.

In urban population, nonsignificant differences were observed for partial edentulous nature in between the female and male subjects as well as for different age groups. It was observed that subjects above 43 years in both female and male were partial edentulous. Female and male subjects, in urban population with partial edentulous, were more in the age group of 43 to 52 years and 53 to 62 years respectively. Among the subjects interviewed and examined, tooth problems were more in the age group of above 43 years compared to the subjects of below 43 years (Table 1). Whereas, in rural population, a signi ficant difference $(<0.05)$ was observed for partial edentulous nature between the female and male subjects, and nonsignificant difference between the different age groups. It was observed that more males (108) were partial edentulous as compared to females (78), and subjects having above 53 years in both female and male were more partial edentulous. Among the subjects interviewed and examined, tooth problems were more in the age group of above 53 years and visiting the hospital in more percentage. In total population, nonsignificant difference (at par) was observed between the female and male subjects for partial edentulous nature as well as between different age groups. Among the subjects interviewed and examined, there were more partial edentulous subjects in both males and females in the age group of above 53 years. More percentage of partial edentulous subjects was observed in females in the age group of above 43 years, while above 53 years in male subjects.

In urban population, nonsignificant differences were observed for complete edentulous nature in between the female and male subjects as well as for different age groups. It was observed that subjects having above 43 years in females and above 53 years in males were more with complete edentulous (Table 1). Whereas, in rural population, a significant difference $(<0.01)$ was observed for complete edentulous nature between the female and male subjects, and nonsignificant difference between the different age groups. It was observed that more males (77.5\%) were complete edentulous as compared to females $(22.4 \%)$, and subjects having above 33 years in male and above 43 years in females were complete edentulous. In total population, nonsignificant difference (at par) was observed between the female and male subjects for complete edentulous nature as well as between different age groups. Among the subjects interviewed and examined, there were more complete edentulous subjects above 33 years in males and above 43 years in females. Whereas, as a whole, subjects above 33 years ( $(98.5 \%)$ in both the sex are complete edentulous.

In total population of dentulous subjects, significant $(<0.01)$ differences were observed between urban and rural living conditions, while nonsignificant for different age groups. It was observed that more urban population was dentulous (206) as compared to rural (127), and subjects of below 53 years in both urban and rural were more dentulous. Among the subjects interviewed and examined, there were no dentulous subjects with above 53 years in both urban and rural conditions (Table 1). 
Table 1: Master chart

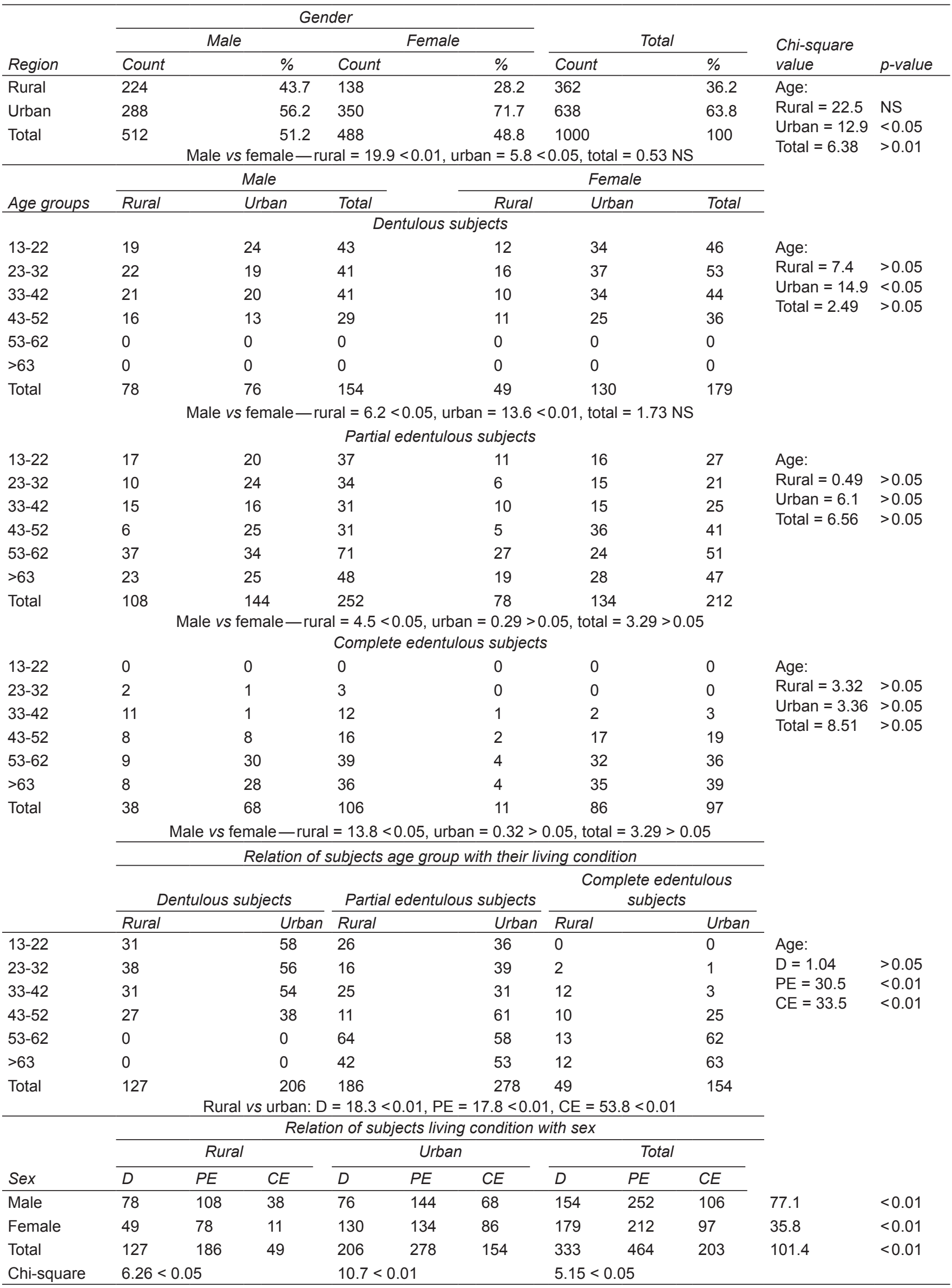

NS: Not significant; D: Dentulous; PE: Partial edentulous; CE: Complete edentulous 
Whereas, in partial edentulous subjects, significant differences $(<0.01)$ were observed for partial edentulous nature between the urban and rural subjects as well as between the different age groups. It was observed that urban population were more partial edentulous (278) as compared to rural (186), and subjects of above 43 and 53 years in urban and rural population showed more partial edentulous respectively. Among the subjects interviewed and examined, there were more partial edentulous subjects (46.8\%) with above 53 years of age. In complete edentulous subjects, significant differences $(<0.01)$ were observed between the urban and rural subjects as well as between different age groups. In urban population, subjects having above 43 years $(97.4 \%)$ were completing edentulous, while above 33 years $(95.9 \%)$ has showed complete edentulous nature in rural population. Among the subjects interviewed and examined, there were more complete edentulous subjects (73.9\%) in total population with above 53 years of age.

In total population of edentulous subjects, significant differences were observed for edentulous nature $(<0.01)$ as well as between different sex groups $(<0.05)$. More partial edentulous subjects (46.4\%) were observed compared to dentulous (33.3\%) and complete edentulous $(20.3 \%)$ subjects (Table 1$)$. The dentulous nature in the subjects were more in females (53.8\%) as compared to males $(46.2 \%)$, while partial and complete edentulism is more in males (54.3 and 52.2\%) compared to females (45.7 and $47.8 \%$ ) respectively. In urban population of edentulous subjects, significant differences $(<0.01)$ were

Table 2: Socioeconomic status and etiology of edentulism

\begin{tabular}{lll}
\hline \multicolumn{3}{c}{ Frequency of etiology causing edentulism } \\
\hline Etiological factors & No. of patients & Percentage \\
\hline Decay & 719 & 71.9 \\
Periodontal disease & 275 & 27.5 \\
Trauma & 5 & 5 \\
Others & 1 & 1 \\
Oral hygiene status & & \\
$\quad$ Good & 197 & 19.7 \\
Fair & 510 & 51.0 \\
Poor & 293 & 29.3 \\
Socioeconomic status of patients & \\
Occupation & & \\
Employed & 463 & 46.3 \\
$\quad$ Unemployed & 537 & 53.7 \\
Monthly family income & & \\
High & 232 & 23.2 \\
Medium & 587 & 58.7 \\
Low & 181 & 18.1 \\
Education & & \\
Illiterate & 207 & 20.7 \\
Basic & 547 & 24.6 \\
Secondary & 246 & \\
\hline
\end{tabular}

observed for edentulous nature as well as between different gender. More partial edentulous subjects $(43.6 \%)$ were observed as compared to dentulous $(32.3 \%)$ and complete edentulous (24.1\%) subjects. The dentulous and complete edentulous nature in the subjects were more in females (63.1 and 55.8\%) as compared to males (36.9 and $44.4 \%$ ) respectively, and partial edentulous is at par in both the sexes. In rural population of edentulous subjects, significant differences $(<0.01$ and $<0.05)$ were observed for edentulous nature as well as between different gender. More partial edentulous subjects (51.4\%) were observed as compared to dentulous (35.1\%) and complete edentulous $(13.5 \%)$ subjects. It was observed that the dentulous, partial and complete edentulous nature in the subjects were more in males $(61.4,58.1$ and $77.6 \%)$ as compared to females (38.6, 41.9 and 13.5\%) respectively.

As shown in Table 2, it was also observed that decay $(71.9 \%)$ was the most common cause of tooth loss in the study population. In addition, based on the oral hygiene status, 510 patients (51\%) belonged to the fair group, 293 patients $(29.3 \%)$ belonged to the poor group and 197 patients (19.7\%) belonged to the good group. It was also observed that decay $(71.9 \%)$ was the most common cause of tooth loss in the study population, as indicated in Table 2. Out of 1000 subjects, 537 patients (53.7\%) were unemployed and 463 patients (46.3\%) were employed. Based on the monthly family income, 587 patients $(58.7 \%)$ belonged to the medium-income group, 232 patients (23.2\%) belonged to the high-income group and 181 patients $(18.1 \%)$ belonged to the low-income group. Distribution based on educational status indicated that 547 patients (54.7\%) belonged to the basic group, 246 patients (24.6\%) belonged to the secondary group, and only 207 patients $(20.7 \%)$ belonged to the illiterate group.

\section{DISCUSSION}

This study is the first one that has evaluated the relationship between age, tooth loss and the frequency of edentulism at Department of Prosthodontics, Government Dental College and Hospital, Indore, India. There are many variables affecting the quality-of-life out of which tooth loss is one of the premier factors. The loss of one or more teeth can have profound effects on an individual's oral health and quality-of-life. ${ }^{19}$

Teeth are required for mastication, phonetics, structural balance and for the comfort of an individual. With the loss of teeth, the above factions are impaired resulting in physical and physiological, psychological trauma to the individual. Further edentulism leads to a deep impact on the quality of life and on physiological and general health status of an individual. ${ }^{19,20}$ Loose teeth, edentulism or ill-fitting dentures may preclude eating 
favorite foods as well as limit intake of favorite foods. Poor oral health and loss of teeth not only affect dietary intake and nutrition status but also compromise general health of the patient. ${ }^{21-23}$

The foremost factors, age and tooth loss both have their variable effects on different age groups affecting the quality-of-life. For an example, in case of infants and small children, tooth loss hampers speech and sucking while in teenagers, esthetics is of major concern, which affects the quality-of-life while performing day-to-day tasks. In addition, along with teeth, several other factors affect the oral health related quality-of-life, the most important among which is personal habit, which includes tobacco usage both in smoking and nonsmoking forms and also alcohol consumption.

According to gender, 512 subjects were males and 488 subjects were females. This tendency for more number of male than female subjects is consistent with some studies, ${ }^{24}$ but not with others. ${ }^{1,2,7,8}$ Suominen-Taipale et al concluded that in 1978 edentulism was found to be more in females than males; but in 1997, the difference between sexes had almost disappeared. ${ }^{2}$ The reason can be attributed to the fact that females were dependent upon the male members of the family to take them for treatment. ${ }^{8}$ Males were not dependent upon any one and they can easily come for treatment.

According to the region, out of 512 male subjects, 224 subjects were from the rural region and 288 subjects were from the urban region. The reasons could be attributed to the fact that there is no healthcare system in rural areas with the dentist population ratio of about 1:200,000. Only $25 \%$ community health centers are having posting of dental surgeons, but have inadequate instruments, equipments and dental materials so they were dependent upon nearby dental colleges for dental treatment. According to the region, out of 488 female subjects, 138 subjects were from the rural region and 350 subjects were from the urban region. This could be because women in the urban region had a better health seeking behavior and were more conscious of their appearance. ${ }^{8}$ Ronald Ettinger and Annette found that people living in urban population (52\%) were least likely to be edentulous than rural population (48\%). ${ }^{25,26}$ More females of urban region (130) were dentulous as compared to males (76), and subjects having below 43 years in both female and male were more dentulous. ${ }^{27}$ Whereas, in rural population, more males (78) were dentulous as compared to females (49), and subjects having below 53 years in both female and male were more dentulous. ${ }^{28}$

Various studies indicated a strong association between age and sex of the patient to edentulism. ${ }^{29-31}$ Nonetheless, contradictory studies refuted positive association between these studied characteristics and partial edentulism. ${ }^{32-34}$ Hence, this study has attempted to elucidate such an association. It was observed that a majority of the study population comprised partially edentulous male patients $(54.3 \%)$ with the peak of the prevalence rate being observed in the age of above 34 years. This study also noted because females were more concern about their oral hygiene and they preferred conservative treatment rather than extraction. ${ }^{35}$

In urban population, complete edentulous female and male subjects were more in the age group of above 52 years and above 43 years respectively, because females were more concern about their oral hygiene and they preferred conservative treatment rather than extraction. ${ }^{35}$ Whereas, in rural population, more males (77.6\%) were complete edentulous as compared to females (22.4\%) subjects having above 33 years in male and above 43 years. ${ }^{36}$

It was observed that more than half of the study population belonged to the medium income group, onefourth of the patients belonged to the high income group and less than one-fourth of the patients belonged to the low income group. Only a small number of patients that approached the outpatient department for treatment belonged to the illiterate group and the majority belonged to the basic education group. It was determined that socioeconomic status is negatively associated with edentulism, with those in lower levels exhibiting higher risks of becoming edentulous. ${ }^{37,38}$ Although, patients belonging to the illiteracy group have more number of missing teeth compared to the other groups, these people belong to socially disadvantaged groups in the society with lack of awareness and financial constraints being the main reason for their neglect. Majority of our study population belonged to the higher education group. This may be attributed to higher levels of income and awareness due to the media and peer group influence. Studies also indicate that patients belonging to the basic educational and low-income levels avail of the resources at the public oral health centers. In addition, it was observed that patients belonging to the higher-income levels approached private oral health clinicians. ${ }^{36,39}$ This explains the reducing number of highly educated people approaching the OPD. Another important observation was that a majority of the study population resided in urban areas. People living in developing countries, especially those living in rural areas, have less access to dental care services when compared to their urban dwelling counterparts. ${ }^{40}$

In regards to oral hygiene status, a majority of the individuals (54.35\%) belonged to the fair group. Edentulous patients tend to have more plaque, suggesting a poorer oral health status. ${ }^{40}$ This could be a direct reflection of low interest in oral healthcare causing subsequent total 
tooth loss. Dental caries and periodontal disease are the two main risk factors for partial tooth loss. ${ }^{40}$ This study noted majority of the teeth lost to be molars which was in accordance with another study conducted by Broadbent et $\mathrm{al}_{1}^{41}$ since the caries experience was greatest in the molar teeth. The highest incidence of caries was noted in the mandibular first molar teeth ${ }^{41}$ since mandibular first molars are the first permanent teeth to erupt in the oral cavity.

\section{LIMITATIONS}

The study was conducted in an institution-based setup. This might bias the study results, as the selected sample is not representative of the state population. Hence, any interpretation of the results of this study must bear this limitation in mind.

\section{CONCLUSION}

Age, gender and tooth loss are associated with each other, but they have independent effects on the oral healthrelated quality-of-life. Tooth loss, which is associated with the increase of age, is associated with more negative impacts while the increase of age independently results in fewer. The necessary step has to be taken for identification and implication of feasible strategies to provide primary dental health education and preventive and rehabilitative treatment to all rural and urban elderly in the future. There is a definite need for a step-by-step approach in eradicating the cause all over the country with special focus on people who suffer from socioeconomic and geographical disadvantage. We suggest community dental health services as a general health need of the elderly rather than a special health need of the community. Incorporating primary as well as rehabilitative dental care services under the existing rural health infrastructure may be an effective method to improve access to oral healthcare for the elderly in developing countries. The consequences of complete edentulism on the oral and facial structures are well-known criteria for predicting the long-term effects of tooth removal on any individual patient. A greater awareness regarding proper dental hygiene and timely replacement of the missing teeth needs to be stressed among the general public.

\section{REFERENCES}

1. Xie Q, Ainamo A. Association of edentulousness with systemic factors in elderly people living at home. Comm Dent Oral Epidemiol 1999;27(3):202-209.

2. Suominen-Taipale AL, Alanen P, Helenius H, Nordblad A, Uutela A. Edentulism among Finnish adults of working age, 1978-1997. Comm Dent Oral Epidemiol 1999;27(5):353-365.
3. Takala L, Utriainen P, Alanen P. Incidence of edentulousness, reasons for full clearance, and health status of teeth before extractions in rural Finland. Community Dent Oral Epidemiol 1994;22(4):254-257.

4. Hunt RJ, Beck JD, Lemke JH, Kohout FJ, Wallace RB. Edentulism and oral health problems among elderly rural Iowans: the Iowa $65+$ rural health study. Am J Public Health 1985;75(10):1177-1181.

5. Slade GD, Spencer AJ. Social impact of oral conditions among older adults. Aust Dent J 1994;39(6):358-364.

6. Agerberg G, Carisser GE. Chewing ability in relation to dental and general health: analysis of data obtained from a questionnaire. Acta Odontol Scand 1981;39(3):147-153.

7. Felton DA. Edentulism and comorbid factors. J Prosthodont 2009;18(2):88-96.

8. Prabhu N, Kumar S, D'Souza M, Hedge V. Partial edentulousness in rural population based on Kennedy's classification: an epidemiological study. J Ind Prosthodont Soc 2009;9(6):18-23.

9. Fure S, Zickert I. Prevalence of root surface caries in 55, 65, and 75-year-old Swedish individuals. Community Dent Oral Epidemiol 1990;18(2):100-105.

10. Shah N, Parkash H, Sunderam KR. Edentulousness, denture wear and denture needs of Indian elderly-a communitybased study. J Oral Rehabil 2004;31(5):467-476.

11. Ariga P, Bridgitte A, Rangarajan V, Philip JM. Edentulousness, denture wear and denture needs of the elderly in rural South India. Iran J Public Health 2012;41(7):40-43.

12. Reddy NS, et al. Epidemiological survey on edentulousness. Contemp Dent Pract 2012;13(4):562-570.

13. Patil VV, Shigli K, Hebbal M, Agrawal N. Tooth loss, prosthetic status and treatment needs among industrial workers in Belgaum, Karnataka, India. J Oral Science 2012;54(4):285-292.

14. Bijjargi S, Chowdhary R. Geriatric dentistry: is rethinking still required? A community-based survey in Indian population. Gerodontol 2013 Dec;30(4):247-253.

15. Shenoy RP, Hegde V. Dental prosthetic status and prosthetic need of the institutionalized elderly living in geriatric homes in mangalore: a pilot study. ISRN Dent 2011:1-3.

16. Kalyanpur R, Prasad KVV. Tooth mortality and prosthetic treatment needs in rural and urban adult population of Dharwad district. Oral Health Prev Dent 2011;9(4):323-328.

17. George B1, John J, Saravanan S, Arumugham IM. Prevalence of permanent tooth loss among children and adults in a suburban area of Chennai. Ind J Dent Res 2011 Mar-Apr; 22(2):364.

18. Lena A1, Ashok K, Padma M, Kamath V, Kamath A. Health and social problems of the elderly: a cross-sectional study in udupi taluk, karnataka. Ind J Comm Med 2009 Apr; 34(2): 131-134.

19. Davis DM, Fiske J, Scott B, Radford DR. The emotional effects of tooth loss: a preliminary quantitative study. Br Dent J 2000 May 13;188(9):503-506.

20. Levin B, Landesman HM. A practical questionnaire for practicing denture success or failure. J Prosthet Dent 1976; 35(2):124-130.

21. Hutton B, Feine J, Morais J. Is there an association between edentulism and nutritional state? J Can Dent Assoc 2002; 68(3):182-187.

22. Geissler CA, Bates JF. The nutritional effects of tooth loss. Am J Clin Nutr 1984;39(3):478-489.

23. Sheiham A, Steele J. Does the condition of the mouth and teeth affect the ability to eat certain foods, nutrient and dietary 
intake and nutritional status amongst older people? Public Health Nutr 2001;4(3):797-803.

24. Sakki TK, Knuuttila ML, Vimpari SS, Hartikainen MS. Association of lifestyle with periodontal health. Comm Dent Oral Epidemiol 1995;23(3):155-158.

25. Bouma J, Schaub RM, van de Poel F. Periodontal status and total tooth extraction in a medium-sized city in the Netherlands. Comm Dent Oral Epidemiol 1985;13(6):323-327.

26. Ettinger RL, Beck JD, Jakobsen J. Removable prosthodontic treatment needs: a survey. J Prosthet Dent 1984;51(3):419-427.

27. Axelsson G, Helgadóttir S. Edentulousness in Iceland in 1990: a national questionnaire survey. Acta Odontol Scand 1995; 53(5):279-282.

28. Ringland C, Taylor L, Bell J, Lim K. Demographic and socioeconomic factors associated with dental health among older people in NSW. Aust NZJ Public Health 2004;28(1):53-61.

29. Shamdol Z, Ismail N, Hamzah N, Ismail A. Prevalence and Associated Factors of Edentulism among Elderly Muslims in Kota Bharu, Kelantan, Malaysia. JIMA 2008;40(4):143-148.

30. Lin HC, Corbet EF, Lo EC, Zhang HG. Tooth loss, occluding pairs, and prosthetic status of Chinese adults. J Dent Res 2001;80(5):1491-1495.

31. Medina-Solís CE, Pérez-Núñez R, Maupomé G, CasanovaRosado JF. Edentulism among Mexican adults aged 35 years and older and associated factors. Am J Public Health 2006;96(9):1578-1581.

32. Downer MC. The improving dental health of United Kingdom adults and prospects for the future. Br Dent J 1991;170: 154-158.
33. Hoover JN, McDermott RE. Edentulousness in patients attending a university dental clinic. J Can Dent Assoc 1989; 55(2):139-140.

34. Marcus PA, Joshi A, Jones JA, Morgano SM. Complete edentulism and denture use for elders in New England. J Prosthet Dent 1996;76(3):260-266.

35. Douglass C, Gillings D, Sollecito W, Gammon M. The potential for increase in the periodontal diseases of the aged population. J Periodontol 1983;54(12):721-730.

36. Shah N, Parkash H, Sunderam KR. Edentulousness, denture wear and denture needs of Indian elderly: A communitybased study. J Oral Rehabil 2004;31(5):467-476.

37. Iacopino AM, Wathen WF. Geriatric prosthodontics: an overview, Part I - pretreatment considerations. Quintessence Int 1993;24(4):259-266.

38. Iacopino AM, Wathen WF. Geriatric prosthodontics: An overview, Part II: Treatment considerations. Quintessence Int 1993;24(5):353-361.

39. Palmqvist S, Söderfeldt B, Vigild M, Kihl J. Dental conditions in middle-aged and older people in Denmark and Sweden: A comparative study of the influence of socioeconomic and attitudinal factors. Acta Odontol Scand 2000;58(3):113-138.

40. Burt BA, Ismail AI, Morrison EC, Beltran ED. Risk factors for tooth loss over a 28-year period. J Dent Res 1990;69(5): 1126-1130.

41. Broadbent JM, Thomson WM, Poulton R. Progression of dental caries and tooth loss between the third and fourth decades of life: a birth cohort study. Caries Res 2006;40(6): 459-465. 